NBER WORKING PAPER SERIES

\title{
INDIVIDUALS' USE OF CARE WHILE UNINSURED: EFFECTS OF TIME SINCE EPISODE INCEPTION AND EPISODE LENGTH
}

Carole Roan Gresenz

Jeannette Rogowski

José J. Escarce

Working Paper 13137

http://www.nber.org/papers/w13137

\author{
NATIONAL BUREAU OF ECONOMIC RESEARCH \\ 1050 Massachusetts Avenue \\ Cambridge, MA 02138
}

May 2007

We thank Sue Polich for programming assistance and Elaine Quiter for project management. We are grateful for helpful comments on an earlier version of this paper from Susan Marquis and participants at the ERIU conference, "Health Insurance: To have and to hold, in sickness and in health?" (September $8,2006)$. The views expressed herein are those of the author(s) and do not necessarily reflect the views of the National Bureau of Economic Research.

(C) 2007 by Carole Roan Gresenz, Jeannette Rogowski, and José J. Escarce. All rights reserved. Short sections of text, not to exceed two paragraphs, may be quoted without explicit permission provided that full credit, including $\odot$ notice, is given to the source. 
Individuals' Use of Care While Uninsured: Effects of Time Since Episode Inception and Episode Length

Carole Roan Gresenz, Jeannette Rogowski, and José J. Escarce

NBER Working Paper No. 13137

May 2007

JEL No. D1,D19,I19

\author{
ABSTRACT \\ second six months of the year compared to the first six months. \\ Carole Roan Gresenz \\ RAND Corporation \\ 1200 South Hayes Street \\ Arlington, Virginia 22202-5050 \\ gresenz@rand.org \\ Jeannette Rogowski \\ Department of Health Systems and Policy \\ University of Medicine and Dentistry of \\ New Jersey (UMDNJ) \\ 335 George Street, Suite 2200 \\ New Brunswick, NJ 08903 \\ and NBER \\ rogowsje@umdnj.edu \\ José J. Escarce \\ RAND \\ 1776 Main Street \\ Santa Monica, CA 90401 \\ escarce@rand.org
}

Few studies have addressed how use of care may vary over the course of an episode of being uninsured or across uninsured episodes of varying duration. This research models the probability that an uninsured individual has (a) any medical expenditures or charges, and (b) any office-based visit during each month of an uninsured episode. We find that the ultimate length of an individual's episode of being uninsured bears relatively little on individuals' use of healthcare in any particular month and that the probability of health care utilization rises during the first year of the episode, with more use in the 


\section{Introduction}

The uninsured are a diverse group, including individuals who lose health insurance coverage for a short period of time and then quickly become insured again, individuals who periodically switch between having and not having health insurance, and those who are persistently uninsured (Monheit and Schur 1988). While a substantial number of studies have analyzed utilization of care among the uninsured, few have addressed how use of care may vary over the course of an episode of being uninsured or across episodes with varying ultimate durations (e.g. short versus long episodes).

Most prior studies of utilization of care among the uninsured rely either on information about individuals who are uninsured at a specific point in time (such as the first day of the calendar year or the day of a survey interview) or on data regarding individuals who are continuously insured or uninsured for a full year (e.g., Cunningham and Kemper 1998; Spillman 1992; Gresenz, Rogowski and Escarce 2006a \& 2006b). A few studies have compared use among individuals with different temporal patterns of being uninsured and found that the short- and long-term uninsured have lower utilization compared to the insured, with use of services generally lowest among individuals who are continuously uninsured (Ayanian et al. 2000; Sudano and Baker 2003; Baker et al. 2001). Other research comparing utilization among individuals with different patterns of insurance over three fourth-month time periods (e.g., insured, uninsured, insured; or 
uninsured, uninsured, insured) finds little evidence that people change their utilization in anticipation of changes in their heath insurance. (Long, Marquis and Rodgers 1998)

Whether and how individuals' use of care varies over the months since the inception of an episode of being without insurance and across uninsured episodes of varying duration are questions this research takes up.

\section{Conceptual Framework}

Individuals' use of care may vary over the course of an episode and for episodes of varying lengths for several reasons. First, it may take time for uninsured individuals to locate, schedule and obtain free or low-cost care. As a result, we may observe lower utilization in earlier months of an episode and greater utilization thereafter among all uninsured.

Second, individuals who are uninsured may attempt to postpone using health care services until they are insured again in order to avoid the high costs of care while uninsured. These costs may include the actual out-of-pocket costs that patients must pay for care as well as the costs associated with the effort of finding low-cost or free care. This hypothesis assumes that at least some care is delayable for some period of time, which is likely to be the case for preventive care, care for self-limited acute conditions, and care for asymptomatic or mildly symptomatic chronic conditions. If individuals have no information about how long their episode of being uninsured will last, they have an 
incentive to try to delay care in the hopes that the period of being uninsured will end before their ability to delay care does. As a result, we are likely to observe a rising probability of care over the months since an episode's inception as individuals' ability to postpone care declines. If everyone is equally uninformed about how long their episode will last, the pattern of rising care over time should not vary across individuals with varying episode lengths, holding all else constant.

On the other hand, if individuals can predict the length of their episode with some degree of certainty, then they may compare the length of time they can delay care to the projected length of the episode. Those with longer episodes will have little or no incentive to delay care (depending on how certain people are about how long they can delay care), while those with shorter episodes will have a stronger incentive to delay care. The result in this case will be that we observe more use of care during a given period of time among the long-term uninsured compared to the short-term uninsured.

However, an alternative possibility is that individuals who are uninsured for short periods of time may use more care compared to the longer-term uninsured. People who believe they will be uninsured for a short period of time may be more likely to use their savings to pay for care compared to those who believe they will be uninsured for a long period of time. The long-term uninsured may guard their savings because of the increased potential for a future serious or catastrophic health need that is not likely to be covered by insurance. 
Generally, the greater the ability of an individual to predict how long their episode will be, the more likely we are to observe differences in use between the long- and shortterm uninsured for a given period of time. Conversely, the weaker the ability of the uninsured to predict episode length, the less likely we are to observe differences in use between the long- and short-term uninsured.

\section{Data and Episode Selection}

We use data from the 1996-2002 Medical Expenditure Panel Survey (MEPS). MEPS is a nationally-representative survey with detailed information on health status, health insurance, and health services utilization. MEPS uses an overlapping panel design in which respondents are interviewed multiple times over a 30-month period to collect data spanning a two year period (Cohen et al. 1996/1997).

We linked the MEPS Household Component (HC) files to MEPS Condition, Event, and Supplemental files. Of particular importance for this study, MEPS-HC data include individuals' monthly health insurance status. The indicators are constructed such that an individual is considered uninsured in a particular month if he/she is uninsured every day of the month. The MEPS Event files record detailed characteristics of individuals' use of care, including date of care. We are thus able to ascribe service use to particular months and to summarize use of care during months in which an individual was uninsured. 
Our sample includes MEPS respondents aged 18-63 who experienced an episode of being without health insurance between 1996 and 2002. ${ }^{1}$ The initial sample includes 24,515 episodes (Figure 1) lasting between 1 and 24 months. Respondents contribute multiple episodes if they fluctuate between being insured and uninsured during the twoyear period in which they are observed. The 24,515 episodes are taken from 22,468 unique individuals, including 20,525 people who had one episode of being uninsured, 1,846 who had two episodes, 90 who had three episodes, and 7 who had four episodes.

We exclude from analysis episodes in which the beginning of the spell is not observed because we are unable to estimate months since inception or episode length for spells in progress. In practice, this means that if an individual were uninsured in the first month of their 24-month observation window, we do not use the episode that included that first month. Of the initial 24,515 episodes, 15,771 were left-censored, leaving 8,744 episodes (Figure 1).

We also exclude from analysis the initial two months of each episode and, consequently, episodes that in their entirety lasted only one or two months. We do this because individuals' recollection of their insurance status may be imperfect, and we found empirical evidence that probable misclassification of insured months as uninsured months was more severe in the first two months of an episode. ${ }^{2}$

As shown in Figure 1, we drop 2,017 episodes that were only one or two months in length, leaving 6,727 episodes in Sample 1. The 6,727 episodes in Sample 1 include 
all episodes in the data whose beginning we observed and that were at least 3 months long. We do not analyze these episodes, but we report their characteristics for comparison with the episodes in our analytic samples, which are described below.

For analysis, we place further limits on the sample. For our main analyses, we exclude episodes that are right-censored (the episode is still in progress at the end of the observation window) if the observed length is less than 12 months. The resulting sample (Sample 2), which includes 4,239 episodes, enables us to assess whether utilization varies between uninsured episodes that last less than one year and those that last one year or longer, but any dependence of utilization on episode length beyond one year cannot be assessed. Therefore, we also conduct sensitivity analyses excluding episodes that are right-censored if the observed length is less than 18 months. This sample (Sample 3) includes 3,141 episodes and enables us to assess whether utilization differs between episodes that last 12-17 months and those that last 18 months or longer.

\section{Specification and Estimation}

The dependent variables are dichotomous indicators for having (a) any expenditures or charges in a month related to office-based, outpatient hospital, or ED care and (b) any office-based physician or non-physician visit in a month. ${ }^{3}$ Each observation is a month period of being uninsured and utilization is measured for the specific observation month. We use multivariate logistic regression analysis to explore how utilization in a given month varies with the number of months since the inception of 
the episode and the ultimate episode length. All regressions were weighted and adjusted for the complex design of the MEPS survey (Cohen et al. 1996/1997).

We control for socio-demographic factors that affect the demand for medical care and detailed measures of health status. These controls are especially important because earlier research shows that individuals in long- versus short-term episodes differ (Swartz and McBride 1990; Swartz, Marcotte and McBride 1993; Short and Friedman 1998; Bennefield 1996). Socio-demographic controls include education, gender, age, genderage interactions, marital status, family size, employment status, race, rural or urban resident, family income as a percentage of the federal poverty line, language of interview, and nativity. We also control for the quarter of the year in which the observed month occurs and include indicators for months that are longer (31 days) or shorter (28 or 29 days) than 30 days.

We measure health status with variables spanning four domains (1) functional, cognitive and social limitations (a single indicator for any such limitation) (2) vision/hearing problems (single indicator for any such problem, including blindness or deafness); (3) self-rated health and mental-health (dichotomous indicators for response categories); and (4) chronic conditions. Using the MEPS Condition files, we constructed indicators for the presence of 25 chronic conditions (such as diabetes, obesity, and asthma) and included specific indicator variables for a subset of those conditions and a summary indicator for the presence of any of the remaining conditions. 
In a first set of regressions, we explore the relationship between episode length and utilization for any given month, without regard to whether the month was near the beginning of the episode, in its middle, or near its end (i.e., without regard to time since inception). These regressions explore whether there is any evidence that utilization throughout the episode is on average higher or lower among the long versus shorter term uninsured. A second set of regressions allow us to see possible variability over time in differences between the uninsured with varying episode lengths—-for example, do we observe that the long-term uninsured use less care in the initial months an episode compared to the short-term uninsured, but more care or the same amount of care in later months of an episode? To answer this type of question, subsequent regressions stratify observations by time since inception: the initial six months of an episode, the $7^{\text {th }}-12^{\text {th }}$ months of the episode, and the $13^{\text {th }}$ through $23^{\text {rd }}$ month of the episode. Only observations from months that fell into these specific periods were included.

In these regressions, we use two alternative specifications for episode length: a dichotomous indicator of whether the episode was less than a year or 12 months or more; and a set of indicators of episode length (for example, 3-4 months long, 5-11 months long, 12 or more months long). For the analyses using the indicator of an episode fewer than 12 compared to 12 or more months long, we exclude only observations where the censored length was less than 12 months (Sample 2). Thus, if an episode was observed to last 14 months but was right censored, it was included because we could definitively classify it as an episode that lasted longer than one year. Likewise, for the analyses that used the series of indicators, we include the observation as long as we can definitively 
classify its length (i.e., as long as a right-censored episode is observed to last at least as long as the greatest length used to classify episodes).

The regressions described allow us to test for statistical differences between utilization in given periods of time across episodes of varying length. We also estimated additional regressions that allow us to test for statistical differences between utilization in an episode of a given length across months since the inception of an episode. Thus, compared to the previous regressions which included controls for episode length and were stratified by time since inception, the additional regressions include controls for months since episode inception and are stratified by episode length. As with episode length, we explored alternative specifications for months since episode inception. We tried dichotomous indicators of 4-month intervals (0-4 months since inception-although only months 3 and 4 are included in analysis, 5-8 months and so on) and 6-month intervals (0-6 months, 7-12 months, etc.). ${ }^{4}$ We ran these regressions for episodes less than 12 months long, 12 or more months long, between 12 and 17 months long, and 18 or more months long. For the stratified regressions with episodes less than 12 months long or more than 12 months long, we used Sample 2, which allows us to definitively classify episodes into one of these groups. For our sensitivity analyses to assess differences between episodes 12-17 months long and 18 or more months long, we used Sample 3.

\section{Results}

\section{Descriptive Data}


Table 1 summarizes characteristics of the various samples used in the analyses. As described earlier and as shown in Figure 1, Samples 1-3 exclude left-censored and short (1 or 2 month) episodes. Sample 2 additionally excludes all episodes that are rightcensored before 12 months, and Sample 3 also excludes all episodes that are rightcensored before 18 months.

While the characteristics of individuals are similar across Samples 1-3, the characteristics of the episodes vary substantially. Sample 1 is the least restrictive sample, with 6,727 episodes. The mean episode length in the sample is 9.1 months. Sample 2 includes 4,239 episodes and 35,911 months for analysis. The mean episode length is 10.5 months. Sample 3 is more restrictive than Sample 2, with 3,141 episodes and 22,474 months and a mean episode length of 9.2 months.

Regressions of Utilization Controlling for Episode Length, with Stratification by Time Since Inception

Table 2 shows results of analyses of utilization and episode length. Columns (i)(iii) are for the dependent variable indicating any expenditures or charges in a month. The column (i) analyses explore whether utilization in any month during the episode is different between individuals with shorter or longer episodes without accounting for which month of the episode the uninsured person is in. By contrast, the column (ii) and (iii) analyses explore, respectively, whether utilization during the third through sixth 
month of an episode and during the seventh through twelfth month of an episode differs by episode length. Columns (iv)-(vi) are parallel to columns (i)-(iii) but are for the dependent variable indicating any office-based visit in a month.

Specification (a) uses a dummy variable indicating whether the episode is more or less than 12 months long. The odds ratios on episode length are not statistically significant for any expenditures or charges in a month or any office-based visits in a month. Specification (b) breaks down shorter episodes into those 3-4 months long versus those 5-11 months long. Again, the odds ratios on episode length are not statistically significant for either dependent variable.

For our sensitivity analyses, specification (c) adds a more detailed categorization of longer episodes by splitting those that are 12 or more months long into those lasting 12-17 months and those lasting 18 or more months. The additional flexibility in specification comes at a price: we can only use observations where we observe either the conclusion of the episode or at least 18 months of it (Sample 3). These analyses include roughly three-fourths of the observations in Sample 2, which was used for Specifications (a) and (b). For both dependent variables, we find no difference in expenditures or visits across episodes of varying length for all months or during the period from 3-6 months since inception. We do, however, find a difference between episodes 12-17 months long and shorter episodes in the probability of utilization for the period between 7 and 12 months since inception (odds ratio $=0.66$ for any expenditures or charges and odds ratio $=0.62$ for any visit). The differences between episodes $12-17$ months long and those 
18 or more months long are also statistically significant $(p<.05$ for any expenditures and any office based visit). In further analyses (not shown), we analyzed the time period from 13-23 months since inception using Sample 3. We found no difference between episodes 12-17 months long and those 18 or more months long.

Regressions of Utilization Controlling for Time Since Inception, Stratified by Episode Length

Table 3 shows results from regressions where we analyzed the relationship between use of care and time since inception for episodes of particular lengths. The upper panel shows results for the any expenditures or charges dependent variable and the lower panel shows results for the dependent variable measuring any office-based visit.

The six-month interval analyses show that for short episodes (less than one year) and longer episodes (greater than one year), the probabilities of any visit and any expenditure are greater in months 7-12 compared to months 3-6. The four-month interval analyses show similar results, suggesting rising utilization during the first year of the episode, for both longer (greater than 12 month) and shorter (less than 12 month) episodes. For intervals after the first year, the odds ratios fluctuate but are not statistically significant, probably as a result of diminishing sample size.

In our sensitivity analyses, we break down longer episodes into those that are medium-length (12-17 months long) and very long (18 or more months long). The sixmonth interval analyses show that for medium- length episodes, the probability of 
utilization is lower in months 7-12 compared to months 3-6 ( $p<.10$ for any visit and any expenditure). The 7-12 versus 3-6 month findings for very long episodes (lasting 18 months or more) are similar to those for episodes 12 or more months long. Similarly, in the four-month interval analyses, utilization in medium (12-17 month) versus very long (18 or more month) episodes appear to have different patterns, although the results are not always statistically significant for both strata.

Figures 2 - 5 show predicted probabilities from the Table 3 models based on Sample 2 using "recycled” predictions. In this type of simulation analysis, all independent variables retain their original values but the month since inception variable (or the interval since inception) is set to a particular value and predictions are calculated for the simulated values. Figures 2 and 3 display predicted probabilities of utilization by six-month and four-month intervals since inception (respectively) for any expenditures or charges. Figures 4 and 5 do likewise for any office-based visit. The figures demonstrate the rising probability of use during the first year of an uninsured episode.

\section{Discussion}

The findings of this study suggest that the ultimate length of an individual's episode of being uninsured bears relatively little on individuals' use of healthcare in any particular month and that the probability of health care utilization rises during the first year of the episode, with more use in the second six months of the year compared to the first six months. Patterns of utilization after the first year of the episode are unclear, as a 
result of limited sample sizes of longer (and uncensored) episodes. One exception to these general findings is from our sensitivity analyses, which found that use of care in some circumstances is lower for medium-length (12-17 months long) episodes compared to very long (18 or more month long) or shorter (less than 12 month long) episodes. The result appears driven by differences during a specific interval (the second half of the first year of the episode).

Putting aside the findings of the sensitivity analyses momentarily, why do we observe such similarities in utilization between short and long episodes? A possible explanation is that individuals are unable to anticipate changes in their insurance coverage. Thus, patterns of care over the course of an episode do not vary among individuals who are near to or distant from gaining insurance coverage because individuals do not know how much longer they will be without insurance. On the other hand, it may be that individuals can anticipate when they will become insured, but either their care is not medically delayable or they do not perceive it to be so. Another possibility is that individuals are myopic in their utilization behavior; they can anticipate changes in their insurance status and know that if they delay care it may be paid for by someone else, but consider only the short-term benefits of receiving the care immediately, and not the longer-term tradeoffs.

The finding that patterns of care over the course of an episode are relatively invariant across short and long uninsured episodes echoes earlier work (Long, Marquis and Rodgers 1998) showing few differences in utilization patterns among individuals 
continuously insured compared to the insured who later lost insurance or among the continuously uninsured compared to those who later gained insurance. These investigators found little evidence that people anticipate changes in their insurance status and modify their health care utilization as a result. Similarly, the RAND Health Insurance Experiment (HIE) analysis of use of care among individuals switching among different insurance plans (Newhouse et al. 1993) found that people did not stock up or spend down their use of hospital and physician services over periods when they were covered by more or less generous plans.

The relationship between utilization and episode length nonetheless warrants further exploration. While we found few statistically significant differences in utilization across episode lengths, the point estimates varied substantially and suggest the potential for differences that might be identified from analyses of larger data sets. Moreover, the findings with regard to medium-length episodes are puzzling and deserve additional attention. Notably, our sensitivity analyses used a smaller and more highly selected sample than the main analyses.

A last reason for continued analysis is that while we have included a comprehensive set of controls for health status, it may still be the case that the health status of individuals with shorter versus longer episodes vary in unobserved ways. If healthier people are less likely to pursue insurance given their more limited needs and have longer episodes, this could confound our ability to measure the particular effect of episode length on utilization. 
Turning to the finding that utilization rises during the first year of the episode, we may observe this pattern of care if it takes time for individuals to locate, schedule and obtain low-cost or free care, resulting in lower utilization in early months. On the other hand, individuals may delay care when they first become uninsured with the hope or knowledge that they will regain insurance at some later date. After the first several months, however, either individuals may not be able or may choose not to delay care further. This explanation, if correct, would lend support to the notion that the invariance in utilization we see among individuals with different episode lengths may result from their inability to anticipate how long they will be uninsured.

Despite the preponderant absence of statistically significant findings regarding episode length and utilization in this research, policymakers may still want to consider policies that distinguish between the short- and long-term uninsured. The long-term uninsured may face health repercussions from the cumulative effect of delaying care for a long period of time as well as significant negative financial consequences of long-term periods without insurance.

It is also worth clarifying that our findings do not imply that the uninsured are getting all the care they need. As previous research suggests, many of the uninsured may go without necessary care and with significant repercussions for their health (e.g., Marquis and Long 1994/95; Hafner-Eaton 1993; Spillman 1992; Hadley, Steinberg and Feder 1991; Lurie et al. 1984). Indeed, one possible reason that we do not observe larger 
transitory shifts in demand for care is that much of the care received by the uninsured is not discretionary. That is, delaying care may be impossible for the uninsured if they are only consuming health care at a level that is at or below the minimum required to maintain some threshold health status. 


\section{Figure 1: Selection of Episodes for Analytic Sample}

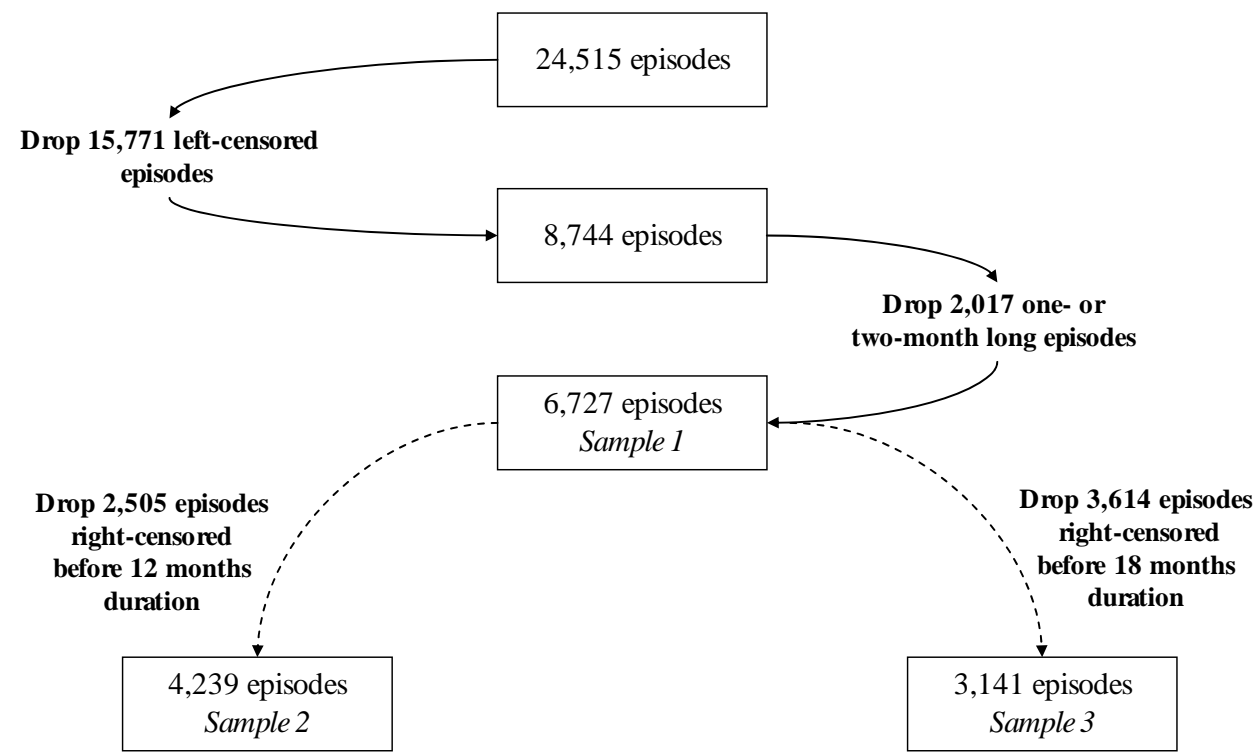


Table 1: Descriptive Statistics for Analytic Samples

\begin{tabular}{|c|c|c|c|c|c|c|}
\hline & \multicolumn{2}{|c|}{ Sample 1} & \multicolumn{2}{|c|}{ Sample 2} & \multicolumn{2}{|c|}{ Sample 3} \\
\hline & \multicolumn{6}{|c|}{ Episodes Excluded } \\
\hline & \multicolumn{2}{|c|}{$\begin{array}{l}\text { Left-censored or } \\
1-2 \text { months long }\end{array}$} & \multicolumn{2}{|c|}{$\begin{array}{l}\text { Sample } 1 \text { plus } \\
\text { right-censored at } \\
\text { less than } 12 \text { months }\end{array}$} & \multicolumn{2}{|c|}{$\begin{array}{c}\text { Sample } 1 \text { plus } \\
\text { right-censored at } \\
\text { less than } 18 \text { months }\end{array}$} \\
\hline Number of episodes & \multicolumn{2}{|r|}{6727} & \multicolumn{2}{|c|}{4239} & \multicolumn{2}{|c|}{3141} \\
\hline Number months for analysis & \multicolumn{2}{|r|}{ N/A } & \multicolumn{2}{|c|}{35911} & \multicolumn{2}{|c|}{22474} \\
\hline Minimum episode length & \multicolumn{2}{|r|}{3} & \multicolumn{2}{|c|}{3} & \multicolumn{2}{|c|}{3} \\
\hline 25th percentile episode length & \multicolumn{2}{|r|}{5} & \multicolumn{2}{|c|}{5} & \multicolumn{2}{|c|}{4} \\
\hline Median episode length & \multicolumn{2}{|c|}{8} & \multicolumn{2}{|c|}{10} & \multicolumn{2}{|c|}{7} \\
\hline 75th percentile episode length & \multicolumn{2}{|r|}{13} & \multicolumn{2}{|c|}{15} & \multicolumn{2}{|c|}{13} \\
\hline 90th percentile length & & 17 & & 9 & & \\
\hline Maximum episode length & & 23 & & 3 & & \\
\hline $\begin{array}{l}\text { Episode begins in 1st quarter of } \\
\text { calendar year (\%) }\end{array}$ & & 17 & & 8 & & \\
\hline $\begin{array}{l}\text { Episode begins in } 2^{\text {nd }} \text { quarter of } \\
\text { calendar year }(\%)\end{array}$ & & 32 & & 0 & & \\
\hline $\begin{array}{l}\text { Episode begins in 3rd quarter of } \\
\text { calendar year (\%) }\end{array}$ & & 29 & & 6 & & \\
\hline $\begin{array}{l}\text { Episode begins in } 4^{\text {th }} \text { quarter of } \\
\text { calendar year }(\%)\end{array}$ & & 22 & & 6 & & \\
\hline & San & ple 1 & & ple 2 & San & le 3 \\
\hline & & Std & & Std & & Std \\
\hline & Mean & Err & Mean & Err & Mean & Err \\
\hline Episode length & 9.07 & $(0.092)$ & 10.49 & $(0.124)$ & 9.18 & $(0.150)$ \\
\hline Income<poverty & 0.18 & $(0.007)$ & 0.19 & $(0.008)$ & 0.18 & $(0.010)$ \\
\hline Income $1-2 x$ poverty & 0.25 & $(0.007)$ & 0.26 & $(0.009)$ & 0.26 & $(0.010)$ \\
\hline Income $2-4 x$ poverty & 0.31 & $(0.009)$ & 0.31 & $(0.010)$ & 0.31 & $(0.013)$ \\
\hline Income $>4 \mathrm{x}$ poverty & 0.26 & $(0.008)$ & 0.25 & $(0.010)$ & 0.25 & $(0.012)$ \\
\hline Less than high school & 0.19 & $(0.007)$ & 0.19 & $(0.008)$ & 0.17 & $(0.009)$ \\
\hline GED or high school diploma & 0.40 & $(0.008)$ & 0.40 & $(0.010)$ & 0.40 & $(0.011)$ \\
\hline Some college & 0.25 & $(0.008)$ & 0.26 & $(0.010)$ & 0.27 & $(0.011)$ \\
\hline College graduate & 0.16 & $(0.007)$ & 0.16 & $(0.008)$ & 0.17 & $(0.010)$ \\
\hline Never married & 0.42 & $(0.008)$ & 0.40 & $(0.010)$ & 0.40 & $(0.012)$ \\
\hline Married & 0.41 & $(0.009)$ & 0.42 & $(0.011)$ & 0.43 & $(0.014)$ \\
\hline Widowed/Divorced/Single & 0.17 & $(0.006)$ & 0.18 & $(0.008)$ & 0.17 & $(0.009)$ \\
\hline Family size & 3.07 & $(0.033)$ & 3.07 & $(0.040)$ & 3.03 & $(0.044)$ \\
\hline Female & 0.53 & $(0.008)$ & 0.54 & $(0.009)$ & 0.55 & $(0.010)$ \\
\hline Aged 18-24 & 0.28 & $(0.008)$ & 0.26 & $(0.010)$ & 0.25 & $(0.012)$ \\
\hline Aged 25-34 & 0.29 & $(0.008)$ & 0.30 & $(0.010)$ & 0.32 & $(0.011)$ \\
\hline Aged 35-44 & 0.23 & (0.008) & 0.23 & $(0.009)$ & 0.23 & $(0.011)$ \\
\hline Aged 45-64 & 0.21 & $(0.007)$ & 0.21 & $(0.009)$ & 0.19 & $(0.009)$ \\
\hline White (non Hispanic) & 0.67 & $(0.010)$ & 0.67 & $(0.011)$ & 0.68 & $(0.012)$ \\
\hline Black (non Hispanic) & 0.14 & $(0.007)$ & 0.14 & $(0.008)$ & 0.13 & $(0.009)$ \\
\hline Hispanic & 0.14 & $(0.006)$ & 0.14 & $(0.007)$ & 0.14 & $(0.008)$ \\
\hline Other minority & 0.05 & $(0.006)$ & 0.05 & $(0.006)$ & 0.05 & $(0.007)$ \\
\hline Interview in English & 0.95 & (0.003) & 0.95 & $(0.004)$ & 0.95 & $(0.005)$ \\
\hline Employed & 0.66 & $(0.008)$ & 0.68 & $(0.009)$ & 0.70 & $(0.010)$ \\
\hline
\end{tabular}




\begin{tabular}{|c|c|c|c|c|c|c|}
\hline US born & 0.82 & $(0.008)$ & 0.82 & $(0.009)$ & 0.82 & $(0.010)$ \\
\hline Missing US born & 0.05 & $(0.004)$ & 0.05 & $(0.005)$ & 0.05 & $(0.006)$ \\
\hline Metropolitan residence & 0.80 & $(0.009)$ & 0.81 & $(0.010)$ & 0.82 & $(0.011)$ \\
\hline Hearing problem & 0.04 & $(0.003)$ & 0.04 & $(0.004)$ & 0.04 & $(0.005)$ \\
\hline Vision problem & 0.04 & $(0.004)$ & 0.05 & $(0.005)$ & 0.05 & $(0.005)$ \\
\hline Functional limitation & 0.06 & $(0.004)$ & 0.06 & $(0.004)$ & 0.06 & $(0.005)$ \\
\hline Social limitation & 0.03 & $(0.003)$ & 0.03 & $(0.003)$ & 0.03 & $(0.004)$ \\
\hline Cognitive limitation & 0.02 & $(0.002)$ & 0.02 & $(0.003)$ & 0.02 & $(0.003)$ \\
\hline Excellent self-rated health & 0.28 & $(0.008)$ & 0.28 & $(0.009)$ & 0.28 & $(0.010)$ \\
\hline Very good self-rated health & 0.34 & (0.009) & 0.34 & $(0.010)$ & 0.34 & $(0.011)$ \\
\hline Good self-rated health & 0.28 & $(0.008)$ & 0.28 & $(0.009)$ & 0.27 & $(0.010)$ \\
\hline Fair self-rated health & 0.08 & $(0.004)$ & 0.08 & $(0.005)$ & 0.08 & $(0.006)$ \\
\hline Poor self-rated health & 0.03 & $(0.002)$ & 0.02 & $(0.003)$ & 0.03 & $(0.003)$ \\
\hline Excellent self-rated mental health & 0.38 & $(0.009)$ & 0.38 & $(0.010)$ & 0.38 & $(0.011)$ \\
\hline Very good self-rated mental health & 0.33 & $(0.008)$ & 0.33 & $(0.010)$ & 0.33 & $(0.011)$ \\
\hline Good self-rated mental health & 0.23 & $(0.007)$ & 0.23 & (0.009) & 0.23 & $(0.010)$ \\
\hline Fair self-rated mental health & 0.05 & $(0.004)$ & 0.05 & $(0.004)$ & 0.05 & $(0.005)$ \\
\hline Poor self-rated mental health & 0.01 & $(0.002)$ & 0.01 & $(0.002)$ & 0.01 & $(0.002)$ \\
\hline Depression & 0.08 & $(0.005)$ & 0.07 & $(0.005)$ & 0.07 & $(0.006)$ \\
\hline Hypertension & 0.08 & $(0.005)$ & 0.07 & $(0.005)$ & 0.07 & $(0.006)$ \\
\hline Diabetes & 0.03 & $(0.003)$ & 0.03 & $(0.003)$ & 0.03 & $(0.003)$ \\
\hline Arthropathies & 0.03 & $(0.003)$ & 0.03 & $(0.003)$ & 0.03 & $(0.004)$ \\
\hline Asthma & 0.04 & $(0.003)$ & 0.04 & $(0.004)$ & 0.04 & $(0.005)$ \\
\hline Non-organic psychoses & 0.04 & $(0.003)$ & 0.04 & $(0.004)$ & 0.04 & $(0.004)$ \\
\hline Migraine & 0.02 & $(0.002)$ & 0.02 & $(0.003)$ & 0.02 & $(0.003)$ \\
\hline Disease of lipid metabolism & 0.03 & $(0.003)$ & 0.03 & $(0.003)$ & 0.03 & $(0.004)$ \\
\hline Other chronic condition & 0.05 & (0.003) & 0.05 & $(0.004)$ & 0.04 & $(0.004)$ \\
\hline Any expenditure or charge & 0.14 & $(0.006)$ & 0.14 & $(0.007)$ & 0.14 & $(0.008)$ \\
\hline Any office-based visit & 0.12 & $(0.006)$ & 0.13 & $(0.007)$ & 0.13 & $(0.008)$ \\
\hline 1996 & 0.16 & (0.009) & 0.22 & $(0.012)$ & 0.20 & $(0.013)$ \\
\hline 1997 & 0.13 & $(0.007)$ & 0.12 & (0.009) & 0.13 & $(0.010)$ \\
\hline 1998 & 0.12 & $(0.008)$ & 0.11 & $(0.008)$ & 0.11 & $(0.009)$ \\
\hline 1999 & 0.14 & (0.009) & 0.14 & $(0.010)$ & 0.15 & $(0.011)$ \\
\hline 2000 & 0.15 & $(0.012)$ & 0.14 & $(0.012)$ & 0.14 & $(0.013)$ \\
\hline 2001 & 0.14 & $(0.007)$ & 0.17 & (0.009) & 0.16 & $(0.009)$ \\
\hline 2002 & 0.14 & $(0.007)$ & 0.10 & $(0.007)$ & 0.12 & $(0.009)$ \\
\hline
\end{tabular}




\section{Table 2:}

\section{Relationship Between Use of Care and Episode Length for All Months and by Months Since Inception}

\begin{tabular}{|c|c|c|c|c|c|c|}
\hline & \multicolumn{3}{|c|}{ Any Expenditures or Charges in a Month } & \multicolumn{3}{|c|}{ Any Office-Based Visit in a Month } \\
\hline & $\begin{array}{c}\text { Any } \\
\text { month } \\
\text { (i) }\end{array}$ & $\begin{array}{c}3^{\text {rd }}-6^{\text {th }} \text { month } \\
\text { since } \\
\text { inception } \\
\text { (ii) }\end{array}$ & $\begin{array}{c}7^{\text {th }}-12^{\text {th }} \\
\text { month } \\
\text { since } \\
\text { inception } \\
\text { (iii) }\end{array}$ & $\begin{array}{c}\text { Any } \\
\text { month } \\
\text { (iv) }\end{array}$ & $\begin{array}{c}3^{\text {rd }}-6^{\text {th }} \\
\text { month } \\
\text { since } \\
\text { inception } \\
\text { (v) }\end{array}$ & $\begin{array}{c}7^{\text {th }}-12^{\text {th }} \\
\text { month } \\
\text { since } \\
\text { inception } \\
\text { (vi) }\end{array}$ \\
\hline \multicolumn{7}{|l|}{ Sample 2} \\
\hline \multicolumn{7}{|l|}{ Specification (a) } \\
\hline Episode less than 12 months long & (ref) & (ref) & (ref) & (ref) & (ref) & (ref) \\
\hline Episode 12 or more months long & 0.99 & 0.98 & 0.86 & 1.01 & 1.03 & 0.87 \\
\hline \multicolumn{7}{|l|}{ Specification (b) } \\
\hline Episode 3-4 months long & (ref) & (ref) & (ref) & (ref) & (ref) & (ref) \\
\hline Episode 5-11 months long & 0.98 & 0.92 & (ref) & 1.04 & 0.97 & (ref) \\
\hline Episode 12 or more months long & 0.97 & 0.92 & 0.86 & 1.05 & 1.01 & 0.87 \\
\hline \multicolumn{7}{|l|}{ Sample 3} \\
\hline \multicolumn{7}{|l|}{ Specification (c) } \\
\hline Episode 3-4 months long & (ref) & (ref) & $\mathrm{n} / \mathrm{a}$ & (ref) & (ref) & $\mathrm{n} / \mathrm{a}$ \\
\hline Episode 5-11 months long & 0.98 & 0.93 & (ref) & 1.05 & 0.99 & (ref) \\
\hline Episode $12-17$ months long & 0.85 & 0.92 & $0.66^{* * *}$ & 0.87 & 0.98 & $0.62 * *$ \\
\hline Episode 18 or more months long & 1.01 & 0.85 & 0.92 & 1.11 & 0.94 & 0.92 \\
\hline
\end{tabular}

Notes: Odds ratios reported. ${ }^{*} p<.10 ;{ }^{* *} p<.05 ; * * * p<.01$. Columns (i) and (iv) include all months of an episode in analyses while the other columns correspond to stratified analyses and hence include only those months in the specified interval. 
Table 3:

\section{Relationship between Use of Care and Months Since Episode Inception by Episode Length}

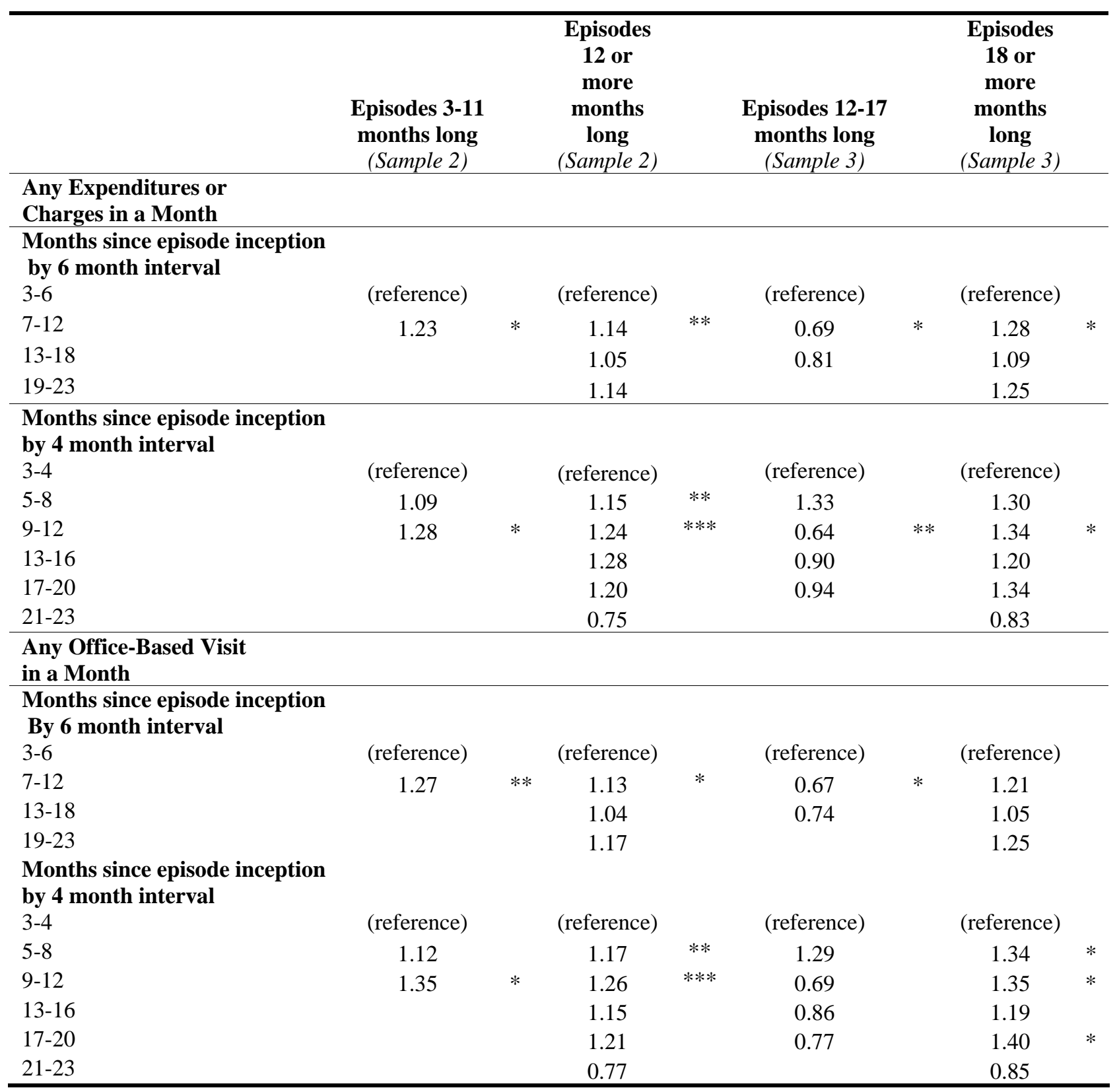

Notes: Odds ratios reported. ${ }^{*} p<.10 ;{ }^{* *} p<.05 ;{ }^{* * *} p<.01$. 
Figure 2:

Predicted Probabilities of Any Expenditure

By Six Month Interval Since Episode Inception, Stratified by Episode Length

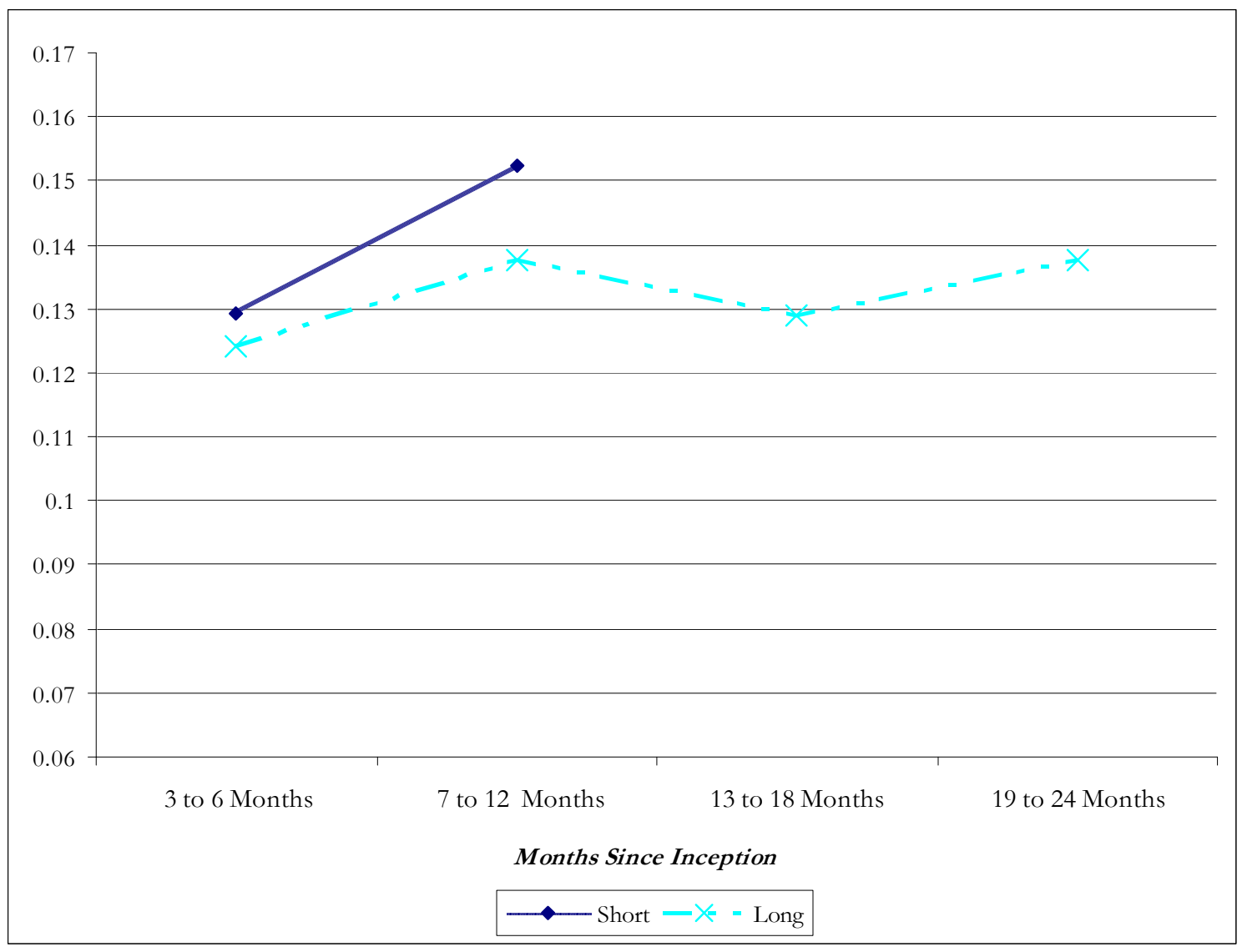


Figure 3:

Predicted Probabilities of Any Expenditure

By Four Month Interval Since Episode Inception,

Stratified by Episode Length

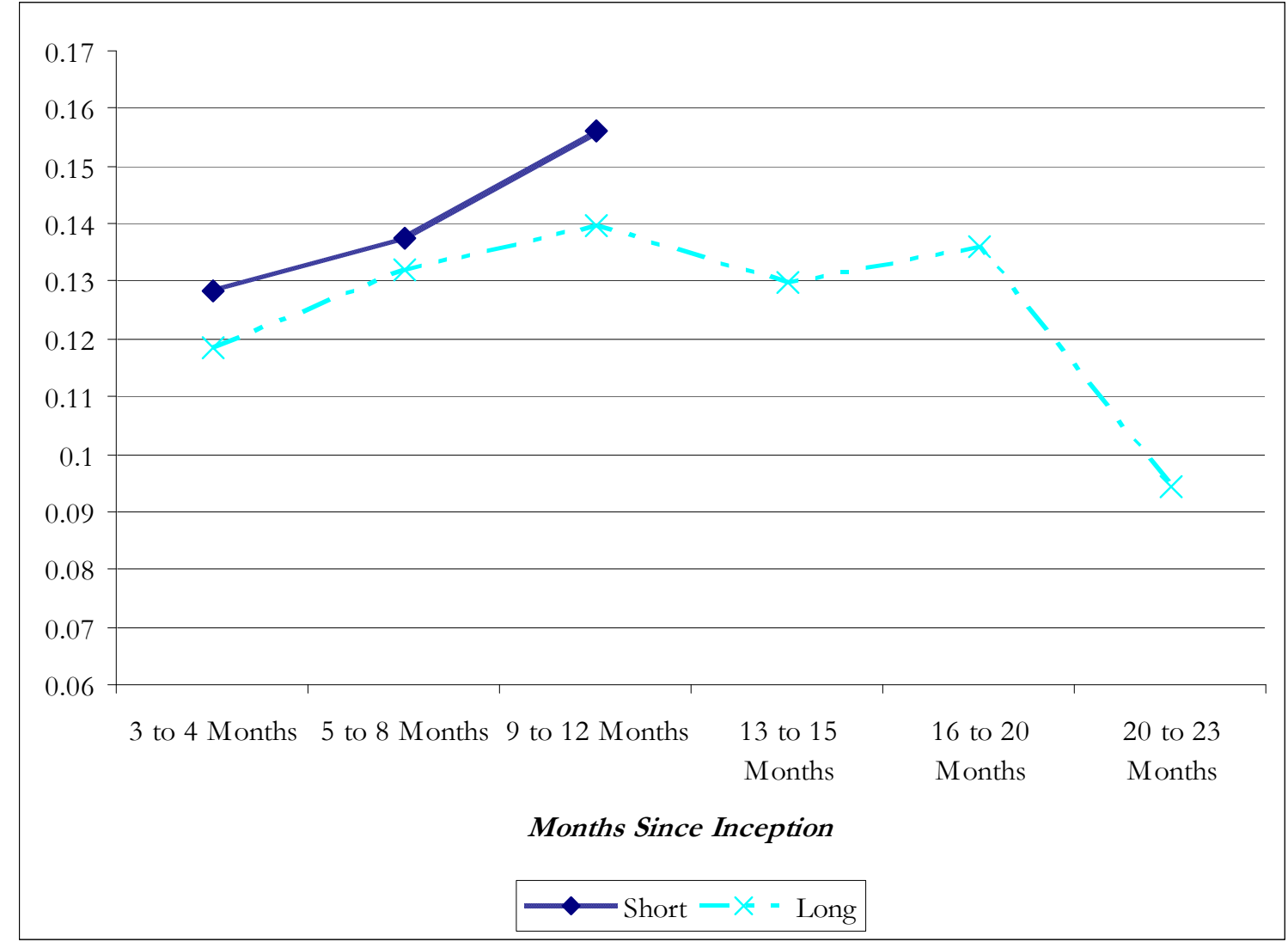


Figure 4:

Predicted Probabilities of Any Office-Based Visit

By Six Month Interval Since Episode Inception

Stratified by Episode Length

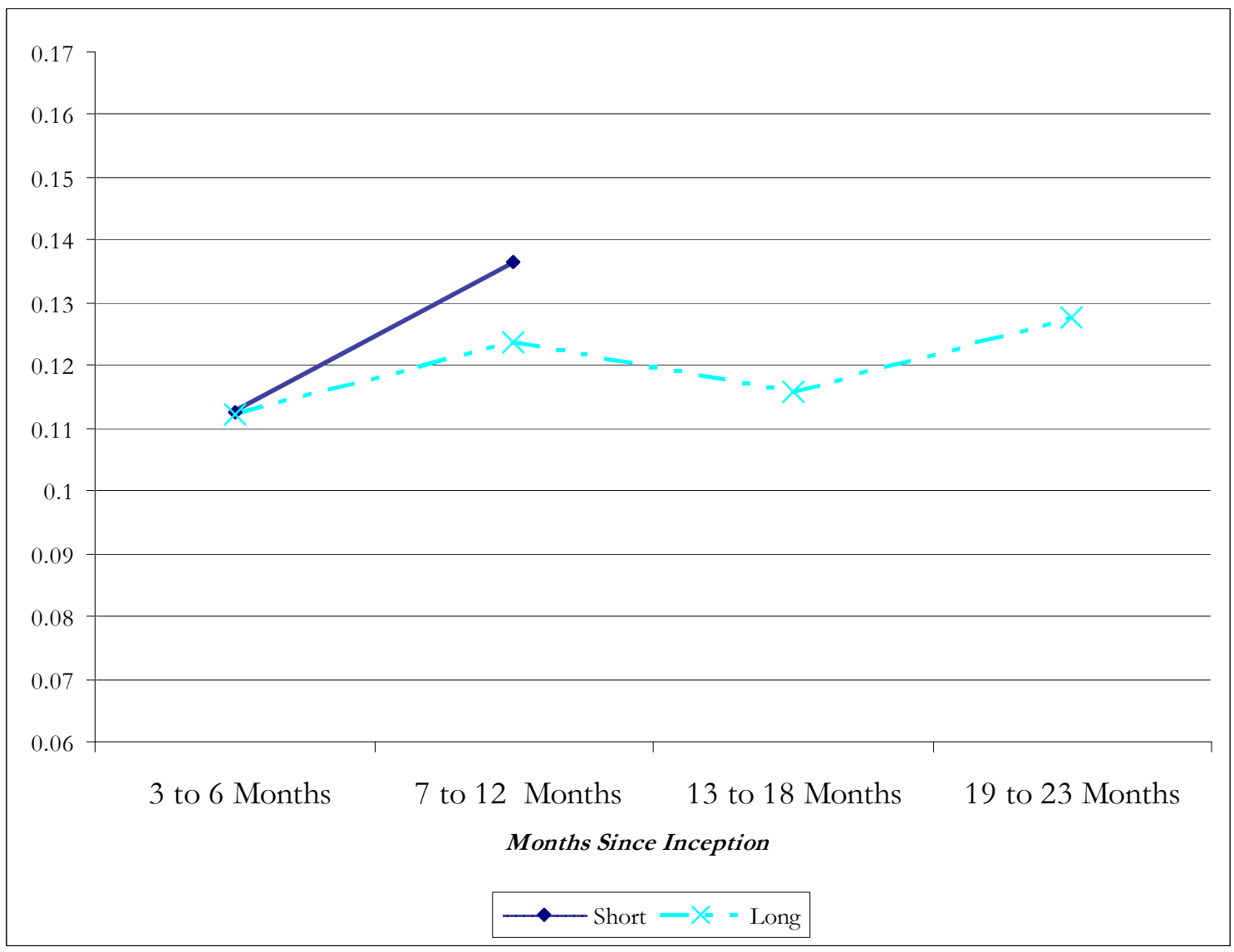

Figure 5: 


\section{Predicted Probabilities of Any Office-Based Visit}

By Four Month Interval Since Episode Inception Stratified by Episode Length

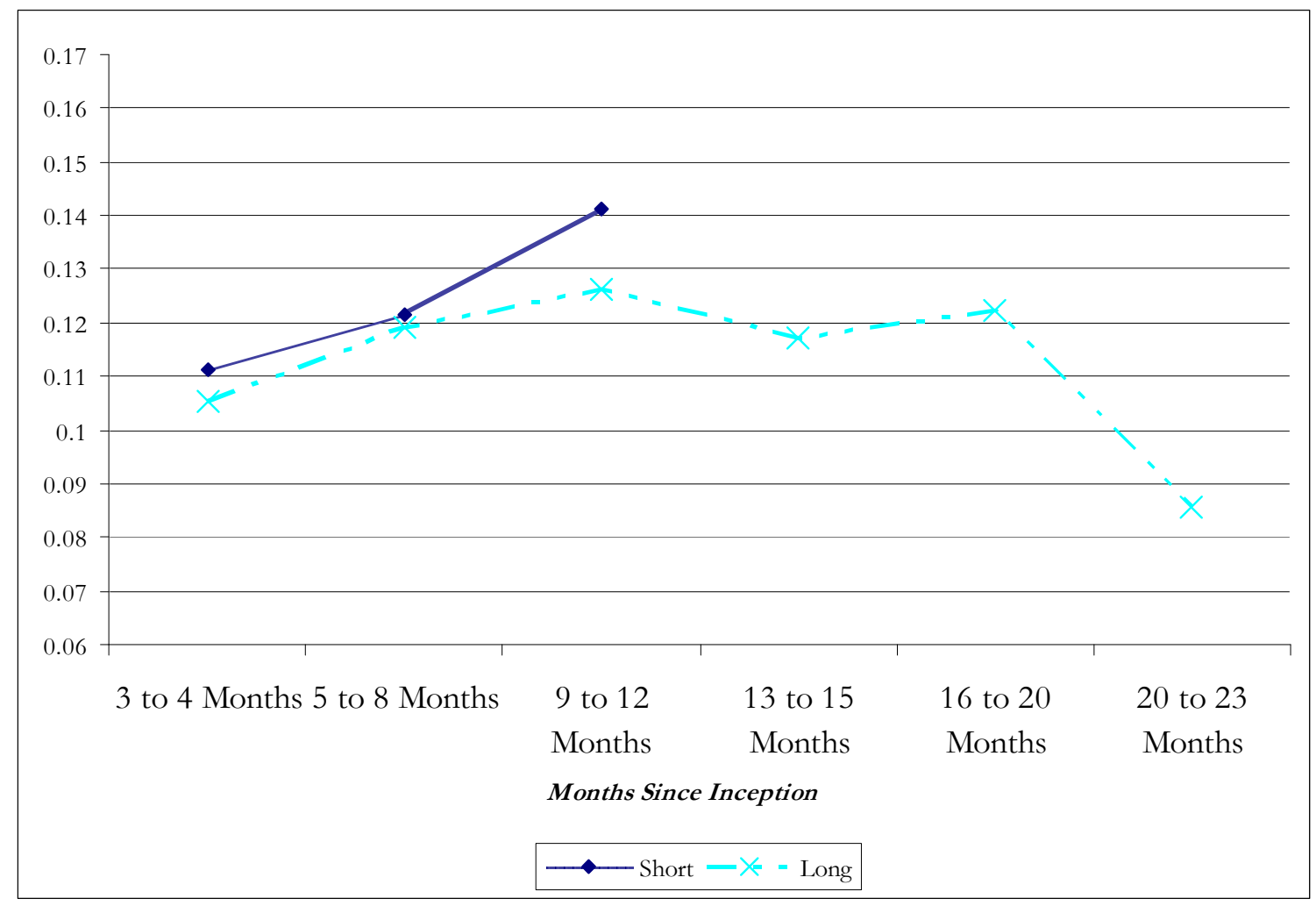




\section{ENDNOTES}

${ }^{1}$ We exclude episodes that end when an individual is 64 years or older. Patterns of utilization as individuals transition from being uninsured to having Medicare at age 65 may differ from patterns of utilization in other episodes because of the virtual certainty of Medicare in contrast to the more uncertain health insurance possibilities in other situations.

${ }^{2}$ For example, 5.4 percent and 4.9 percent of individuals in their first- and secondreported months of an uninsured episode, respectively, had at least some expenditures paid for by insurance, compared to an average of 3.9 percent of individuals in months 312. Among those reporting at least some expenditures in the first two months of their episode, more than one-fourth (26.1\% in month 1 and $26.3 \%$ in month 2 ) reported that a significant fraction ( $80 \%$ or more) of their expenditures were paid for by some private source, compared to an average of less than $20 \%$ who reported a significant fraction of privately paid expenditures in months 3-12.

${ }^{3}$ Prescription expenditures and other medical expenditures (such as for durable medical equipment) cannot be apportioned to specific months. These expenditures as well as dental, vision and inpatient hospital expenditures and charges are excluded.

${ }^{4}$ We note that while we excluded the first two months of each episode from analysis, our measure of months since inception is from the reported inception. We could have 
alternatively subtracted two months from each of our measure of months since inception when labeling those variables. 


\section{REFERENCES}

Ayanian, J.Z., J.S. Weissman, E.C. Schneider, J.A. Ginsburg, A.M. Zaslavsky, 2000. Unmet Health Needs of Uninsured Adults in the US. Journal of the American Medical Association 284:2061-2069.

Baker, D. W., J.J. Sudano, J.M. Albert, E.A. Borawski, A. Dor. 2001. Lack Of Health Insurance And Decline In Overall Health In Late Middle Age. New England Journal of Medicine 345:1106-1112.

Bennefield, R. L. 1996. Dynamics of Economic Well being: Health insurance 19921993: Who loses coverage and for how long? Current Population Reports 70-54 (May): 1-5.

Cohen JW, Monheit AC, Beauregard KM, Cohen SB, Lefkowitz DC, Potter DEB, et al. The Medical Expenditure Panel Survey: A national health information resource. Inquiry. 1996/1997;33:373-89.

Cunningham, P. J. and P. Kemper. 1998. Ability to Obtain Medical Care for the Uninsured: How Much Does It Vary Across Communities? Journal of American Medical Association 280 (10): 921-927. 
Gresenz, C.R., J. Rogowski, and J. Escarce, Dimensions of the Local Health Care Environment and Use of Care By Uninsured Children in Rural and Urban Areas, Pediatrics 117:509-517, 2006a.

Gresenz, C.R., J. Rogowski, and J. Escarce. Healthcare Markets, the Safety Net, and Utilization of Care Among the Uninsured. Health Services Research, forthcoming, 2006b.

Hadley, J., E.P. Steinberg, and J. Feder. 1991. Comparison of Uninsured and Privately Insured Hospital Patients: Condition on Admission, Resource Use, and Outcome. Journal of the American Medical Association 265:374-79.

Hafner-Eaton, C. 1993. Physician Utilization Disparities Between the Uninsured and Insured. Journal of the American Medical Association 269:787-92.

Long, S. H., M. S. Marquis, and J. Rodgers. 1998. Do People Shift Their Use of Health Services over Time to Take Advantage of Insurance? Journal of Health Economics 17: 105-115.

Lurie, N., N. B. Ward, M. F. Shapiro, and R. H. Brook. 1984. Termination from MediCal: Does it Affect Health? New England Journal of Medicine 311:480-84. 
Marquis, M.S., and S.H. Long. 1994/1995. The Uninsured Access Gap: Narrowing the Estimates. Inquiry 31:405-14.

Monheit, A.C. and C.L. Schur. 1988. The Dynamics of Health Insurance Loss: A Tale of Two Cohorts. Inquiry 25: 315-327.

Newhouse, J.P. and the Insurance Experiment Group (R.W. Archibald, H.L. Bailit, R.H. Brook, M. Brown, A.R. Davies, N. Duan, G.A. Goldberg, E.B. Keeler, A. Leibowitz, K.N. Lohr, W.G. Manning, Jr., K.H. Marquis, M.S. Marquis, C.N. Morris, C.E. Phelps, W.H. Rogers, C.A. Sherbourne, R. Burciaga Valdez, J.E. Ware, Jr., K.B. Wells) 1993. Free for all? Lessons from the RAND Health Insurance Experiment. Cambridge, MA: Harvard University Press.

Short, P.F. and V.A. Freedman. 1998. Single Women and the Dynamics of Medicaid. Health Services Research 33(5): 1309-1336.

Spillman, B. C. 1992. The Impact of Being Uninsured on Utilization of Basic Health Care Services. Inquiry 29: 457-466.

Sudano, J.J. and D.W. Baker. 2003. Intermittent Lack of Health Insurance Coverage and use of Preventive Services. American Journal of Public Health 93(1): 130-137.

Swartz, K. and T. D. McBride. 1990. Spells without Health Insurance: Distributions of 
Durations and Their Link to Point-in-Time Estimates of the Uninsured. Inquiry 27: 281288.

Swartz, K., J. Marcotte, and T. D. McBride. 1993. Spells without Health Insurance: The Distribution of Durations When Left-Censored Spells are Included. Inquiry 30: 77-83. 